\title{
Tracing the Change in Discourse in a Collaborative Dynamic-Geometry Environment: From Visual to More Mathematical
}

\author{
Diler Oner and Gerry Stahl
}

In this paper, we investigate what appears to be an intermediate developmental stage in geometrical thinking according to Sfard's commognitive framework. More specifically, we examine the change in three middle-school students' mathematical discourse as they worked on a geometry construction problem within a virtual collaborative dynamic-geometry environment. We trace how their word use, use of visual mediators, and routines changed character during an hour-long collaborative problem-solving session. Our findings indicate that students gradually moved from a visual towards a more formal discourse -- one that is primarily characterized by a routine of constructing dependencies. We conclude that interacting with expert interlocutors may not be the only path towards advancing one's mathematical discourse; this process may also take place within a computer-supported collaborative-learning setting. Thus, collaborative dynamic geometry can be viewed as an effective material condition for learning geometry.

Key words: mathematical discourse; collaborative dynamic geometry; dependencies; middle school

\section{CSCL Support for Mathematical Development}

Students' geometrical thinking development has been an important area of study for researchers. In particular, how learners make the transition towards formal mathematics has attracted much interest. In the context of dynamic-geometry environments (DGEs), the more formal is usually associated with the ability of constructing figures. There is a distinction between a drawing and a construction within a DGE. Drawing refers to the juxtaposition of geometrical objects that look like some intended figure (Hoyles \& Jones, 1998). Construction, however, depends on creating relationships, in other words dependencies (Stahl, 2013a), among the elements of a figure. Once relationships are defined and constructed accordingly, the figure maintains these theoretical relationships even under dragging.

The transition from visual towards formal mathematics, nonetheless, has been found to be neither straightforward nor easy for students working with dynamic geometry (Jones, 2000; Marrades \& Guttierez, 2000). Students often think that it is possible to construct a geometric figure based on visual cues (Laborde, 2004). One can then make distinctions between two different mathematical discourses (Sfard, 2008) in which students may engage when working within DGEs. Within one of these, students may talk about geometrical figures as if they are merely visually perceptual entities -- without making any connections between them and theoretical relationships they signify. When presented with a geometry construction problem, students might adopt a solution routine (ibid.) that is based on visual placement and verification, which produces a drawing (Hoyles \& Jones, 1998). Taking a more sophisticated mathematical discourse, however, they would frame the problem as construction, that is, one that involves defining dependencies (Stahl, 2013a).

Sfard argues that such a discursive jump to more sophisticated discourse takes place "while participating in the discourse with more experienced interlocutors" (p. 191). However, we have found that participation within a computer-supported collaborative learning setting can also help students move forward from visual towards more formal ways of dealing with a construction problem. The setting we have explored is the Virtual Math Teams (VMT) environment. The VMT is an open-source, virtual, collaborative learning environment that affords synchronous text-based interaction (chat) with an embedded multi-user dynamic-geometry application, GeoGebra. VMT is regarded as the first sustained effort supporting a collaborative form of dynamic geometry (Stahl, 2013a).

In this paper, we investigate how three middle-school students, who initially treated a geometry construction task visually, moved towards more mathematical ways of approaching the problem while working within the VMT environment. In other words, we map out an intermediate state in which the students, who had very limited formal geometry background, were in transition from one mathematical discourse to another. By doing that, we argue that interacting with expert interlocutors may not be the only path towards advancing one's mathematical discourse. We suggest that this process may also take place within a virtual collaborative setting where dynamic 
geometry, collaboration, and task instructions collectively fulfill a role similar to that of the discourse of experts. Better understanding how that transition was made and the characteristics of the intermediate stage will help in designing more effective tasks and approaches for DGEs.

\section{Theoretical Framework}

Drawing from Sfard (2008), we take mathematics as discourse and learning as development of discourses. Sfard frames (mathematical) thinking as an individualized form of communication. Thus, she suggests a developmental unity between the processes of communicating and thinking, which leads to naming her approach commognitive. Commognitive researchers are interested in mathematical discourses, as this is where one can trace the processes of learning. Sfard distinguishes mathematical discourses in terms of their tools, words and visual means, and the form and outcomes of their processes, routines and narratives. We explain each of these constructs below but mainly focus on the notion of routines, as it is the most relevant for the analysis in this study.

Different mathematical discourses employ particular mathematical words, which might signify different things in different discourses, and visual objects, such as figures or symbolic artifacts. In addition to these discourse tools, participants functioning in different discourses produce what Sfard calls narratives, that is, sequences of utterances about mathematical objects and relations among them. Narratives are subject to endorsement or rejection under certain substantiation procedures by the community. Endorsed narratives usually take the form of definitions, axioms, theorems, and proofs. In order to produce mathematical narratives, participants engage in mathematical tasks in specific ways. They follow what are called metarules, which are different than object-level rules. Rules that express patterns about mathematical objects, say about triangles, are defined as object-level rules (e.g., the sum of interior angles of a triangle is $180^{\circ}$ ). Metarules, on the other hand, are about actions of participants, and they relate to the production and substantiation of object-level rules. The set of metarules that describes a patterned discursive action are named routines, since they get repeated in certain types of situations.

Routines take two forms: the how and the when of a routine. The how of a routine, which may be called a procedure, refers to a set of metarules describing the course of the patterned discursive action. The when of a routine, on the other hand, is a collection of metarules used by participants to determine the appropriateness of the performance. The researcher might observe the how of a routine more easily when $\mathrm{s} / \mathrm{he}$ assigns a specific task. Examining the when of a routine, however, requires extended periods of observations (weeks, months, or more) when participants are asked to solve more complex problems. In this study, given that students were provided with a well-defined task, the how of a routine was analyzed.

Sfard (2008) states that metadiscursive rules and routines are the researcher's construct based on observations of participants' discursive actions. Therefore, they are about the past. However, they are useful constructs for the researcher as "[c]onstructed metarules allow us to map the trajectory of one's discursive development" (p. 209).

\section{The Context and Participants}

The data come from a team of three eighth-grade students (about 14 years old) who worked on a geometry construction problem collaboratively within the VMT environment. We called them the Cereal team, because they named themselves Cheerios, Cornflakes, and Fruitloops. None of the team members had studied geometry, but they were taking first-year algebra at the time of data of collection. They were all females. Before this session, they met within VMT for two hour-long sessions, trying basic GeoGebra tools, such as the software tools for creating points, lines, and line segments, and working on the task of equilateral-triangle construction.

The task given to the students comes from a geometry curriculum written by Stahl (2013b) for the VMT environment. It is named Topic 3, the third topic within that curriculum. The task presented in Topic 3 had two parts. In the first part, the team was asked to construct two lines, which are perpendicular bisectors of each other. A list of steps was provided so that students would construct the diagonals (AB and $\mathrm{CD}$ ) of a rhombus (ACBD) and its diagonals. A completed construction was provided (Figure 1a) as an illustration. The second part of the task asked students to construct a perpendicular line to a given line through a given point. Here, one first needs to define the given point as a midpoint between two points using the circle tool and then follow the same steps with the rhombus construction (Figure 1b). As simple as it sounds, however, given our participants' limited geometry background, this task was not trivial for the Cereal team.

Participants work on geometry problems in the VMT environment within specific chat rooms created for each topic. Figure 1a shows the VMT room created for Topic 3 with a chat (on the right hand side) and a 
whiteboard area where multi-user GeoGebra is integrated. One can post a chat anytime during the session.

However, in order to use the GeoGebra area one has to click on the 'take control' button (at the bottom). Thus, only one person at a time can use the dynamic geometry section of the room. The GeoGebra view is, however, shared by everyone in the team, so they can all observe changes to the figures as they are made.

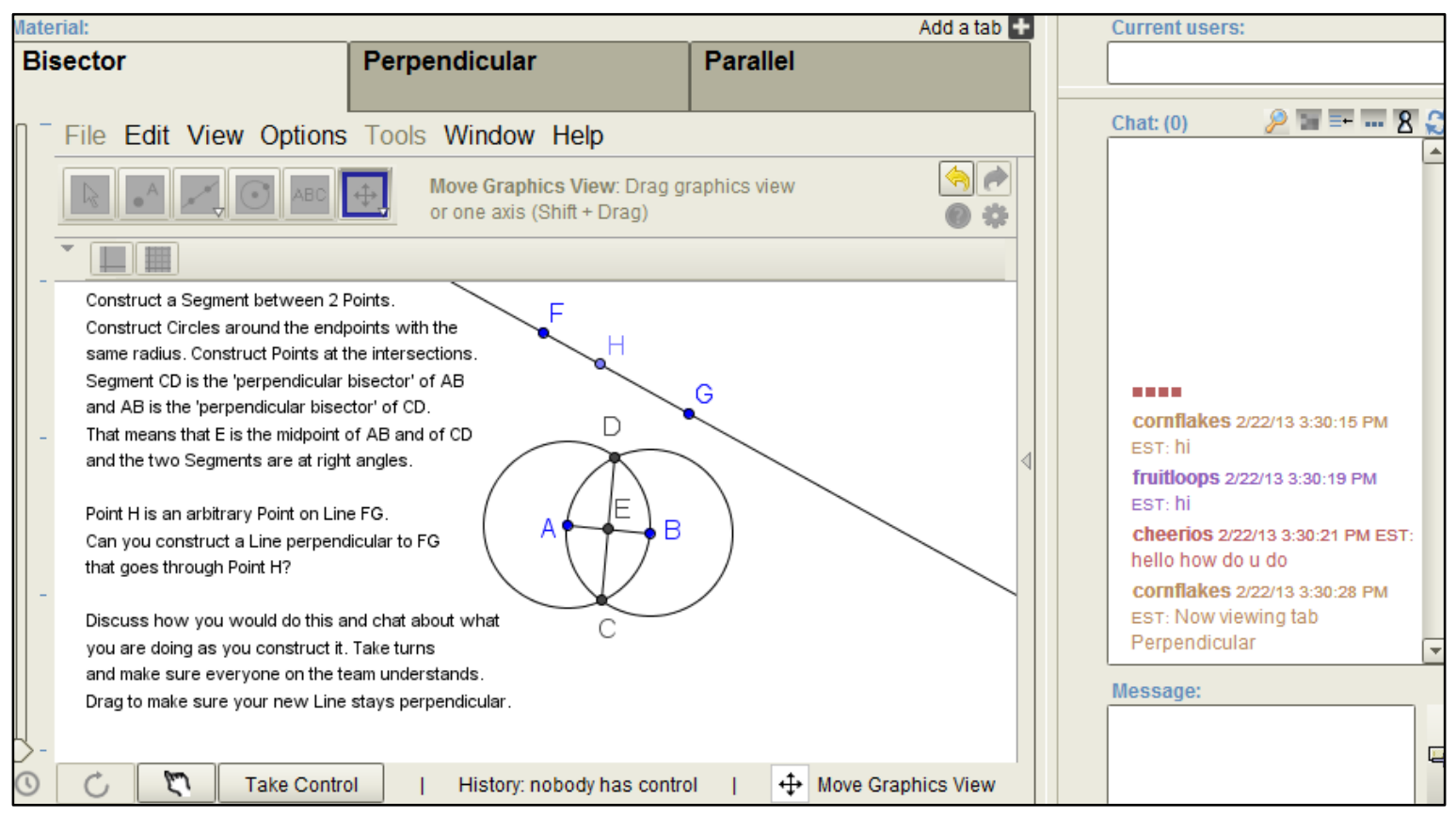

Figure 1a. The VMT room near the beginning of work on Topic 3.

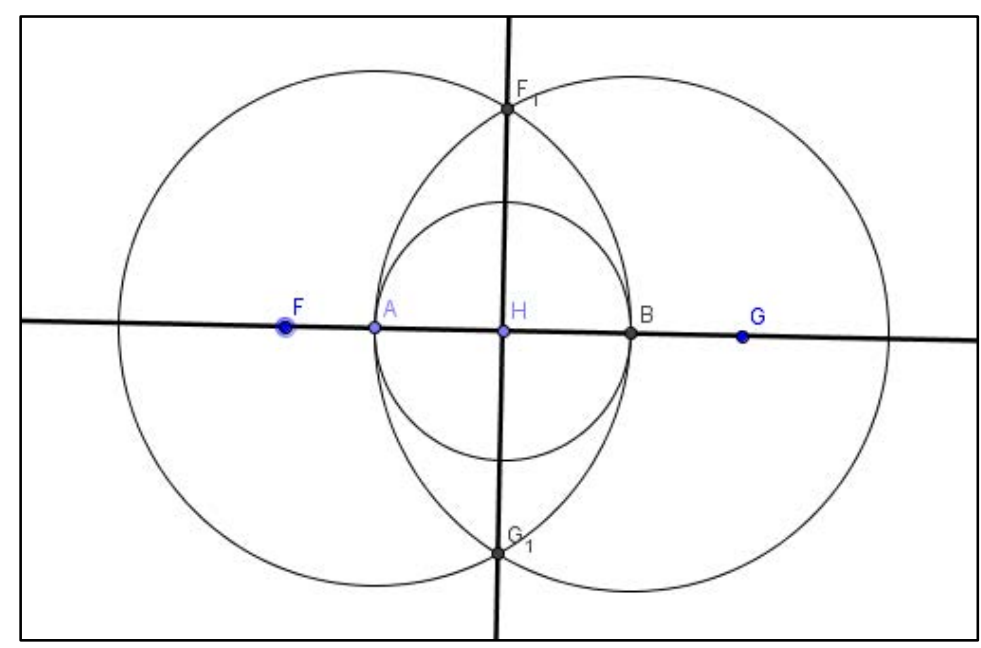

Figure $1 \mathrm{~b}$. The expected construction of the perpendicular to the line FG through a given point $\mathrm{H}$

\section{Data Collection and Analysis}

The team's meeting over the VMT environment had been arranged as part of an after-school club by their math teacher. The Cereal team worked on Topic 3 for about an hour. The problem-solving session has been recorded as a VMT log file to be replayed later, allowing the observation of the team's problem-solving process in micro-detail. All chat postings and GeoGebra actions produced by the team members were recorded and marked by the individual colors assigned to each member. 
In order to investigate the changes in participants' discourse, we examined both the chat postings and the actions of the participants recorded in their VMT session through Sfard's (2008) discursive lens. We particularly focused on the changes in (1) their use of the word "perpendicular," (2) the visual mediators they acted upon, and (3) their routines, as the changes in these features were the most salient aspects of their changing discourse. Given the nature of the task, this study investigated two routines: (a) the production of the perpendicular and (b) the verification of perpendicularity.

The production of the perpendicular routine involved the use of a set of procedures referring to the repetitive actions in producing a perpendicular line. We observed two contrasting production routines: (i) visual placement by drawing or dragging and (ii) construction by creating objects with dependencies between them. The verification of perpendicularity routine is a set of procedures describing the repetitive actions in substantiating whether a solution (a line produced) is in fact perpendicular to a given line. We observed two contrasting verification procedures: (i) visual judgment or measurement, and (ii) use of theoretical geometry knowledge to justify proposed solutions.

Two discourses are considered distinct when they are incommensurable, that is, when they have different rules for the same type of task (Sinclair \& Moss, 2012). We thus distinguish between two mathematical discourses when they entailed different ways of solving the task in our context. In one of these, students' production of the perpendicular and verification of perpendicularity are exclusively based on spatio-graphical cues without any concern for theoretical relationships. More specifically, the solution and verification routine is based on visual placement of a perpendicular-looking line (spatio-graphical solution), which produces a drawing (Hoyles \& Jones, 1998). The use of the word "perpendicular" also reflects a visual image in which two lines perceptually look perpendicular. Thus, we refer to this discourse as visual. In another discourse, which we call formal, the production of the perpendicular line involves constructing dependencies, that is, defining relationships using the software tools. The verification routine within this discourse is theoretical deriving from geometrical relationships. The word "perpendicular" within this discourse signifies a theoretical relationship between geometrical objects.

As the first step in the analysis, the chat postings and GeoGebra actions of the Cereal team were divided into episodes mainly based on the detected changes in participants' routines of solving the task (i.e., routines of production and verification). In each episode, what is said and done were examined focusing on the three aspects of their mathematical discourse when relevant: their use of the word "perpendicular," the visual means acted upon, and routines of the production of the perpendicular and verification of perpendicularity in each episode. In the next section, we summarize our analysis of these episodes.

\section{Results and Discussion}

Based on the team's routines of production and verification, the interaction was divided into the following episodes: (1) constructing the perpendicular bisector, (2) drawing a perpendicular-looking line (3) drawing the perpendicular using the perpendicular bisector construction (PBC) as straightedge, (4) use of circles with no dependencies defined, (5) constructing dependencies, and (6) discussing why the construction worked. We saw that the team started constructing two line segments as perpendicular bisectors of each other following the specific instructions. In this part, Cheerios' use of the word "perpendicular" was copied from the instructions as if using a foreign language word in a sentence. They next moved to the second task, which was built on the first one, yet presented at least two challenges for them. First, the team needed to figure out how to construct a perpendicular to a line through a given point, which they had not done before. In the Table 1 below, we summarize their use of the word perpendicular, their use visual mediators, and routines of production of a perpendicular and verification of perpendicularity in the rest of episodes where the team mainly focused on the second task in Topic 3.

Table 1 . The change in discourse in Topic 3 (summary)

\begin{tabular}{|c|c|c|c|c|}
\hline Episode & $\begin{array}{l}\text { Production of the } \\
\text { perpendicular routine }\end{array}$ & $\begin{array}{l}\text { Verification of } \\
\text { perpendicularity routine }\end{array}$ & $\begin{array}{l}\text { Use of the word } \\
\text { perpendicular }\end{array}$ & $\begin{array}{l}\text { Use of visual } \\
\text { mediators }\end{array}$ \\
\hline 2 & $\begin{array}{l}\text { Creating another reference line in } \\
\text { relation to line FG (Cornflakes } \\
\text { and Cheerios) }\end{array}$ & & $\begin{array}{l}\text { Signifying a visual } \\
\text { image of } \\
\text { perpendicular to } \\
\text { disagree with a } \\
\text { spatio-graphical } \\
\text { solution }\end{array}$ & \\
\hline
\end{tabular}


Spatio-graphical solution / drawing a perpendicular-looking line (Cornflakes)

Spatio-graphical solution / drawing a perpendicular-looking line (Cheerios \& Cornflakes)

Spatio-graphical solution (Cornflakes, Fruitloops, Cheerios)

3

Spatio-graphical solution / imitation of paper-pencil routine of drawing the perpendicular using PBC as straightedge (Fruitloops \& Cornflakes)

4

Use of circles with no dependencies defined (Fruitloops)

5

-Constructing dependencies / use of equal-radius circles (Fruitloops)

-Dynamic solution / attaching the arbitrary point $\mathrm{H}$ to the line (Fruitloops)
PBC-random dragging (Cornflakes)

Signifying a visual image of perpendicular to disagree and then agree with a spatiographical solution (Fruitloops)

Spatio-graphical verification / vertical-horizontal alignment of the lines (Cheerios)

Signifying a visual image of perpendicular to agree with a spatiographical solution (Fruitloops)

Measurement-based verification using $\mathrm{PBC}$ (Cornflakes \& Fruitloops)

-PBC as protractor (Cornflakes)

-PBC as straightedge (Fruitloops)

PBC as image of construction (Fruitloops)
6

- Spatio-graphical
(Cornflakes)
-Looking for a verification
routine beyond spatio-

Signifying a mathematical relationship (Fruitloops)
Signifying a mathematical relationship (Fruitloops) 
graphical evidence

(Fruitloops \& Cornflakes)

If we look at the change in the production of the perpendicular routine column in Table 1, we see that the team started with producing spatio-graphical solutions including placing the perpendicular line visually and imitation of the paper-pencil procedure of drawing the perpendicular by using the $\mathrm{PBC}$ as a straightedge guide. These routines however evolved into first the use of circles and then defining certain relationships with the circles, such as use of equal-radius circles with the construction, leading the group to successfully completing the task. Thus, the team's work within the visual discourse mode laid a basis for conceptualizing the task in a more formal discourse mode. One discourse evolved into the other.

The second dependency (having the perpendicular pass through point $\mathrm{H}$ ), however, was bypassed by simply dragging the arbitrary point $\mathrm{H}$ to the perpendicular line. That is, the Cereal team's final solution did not involve taking the arbitrary point $\mathrm{H}$ as the reference point for constructing the perpendicular line. The instructions specified that "point $\mathrm{H}$ is an arbitrary point on line FG." In Euclidean geometry, that means that even though H can be any point on line FG, it is not something that moves. Thus, although one looks for a solution that would work for any point $\mathrm{H}$, any treatment of $\mathrm{H}$ would be static. In dynamic geometry, however, an arbitrary point $\mathrm{H}$ is a free point that can be dragged along line FG. Furthermore, the team's solution (dragging the point $\mathrm{H}$ to the perpendicular line) did not fail the drag test. For these reasons, we consider their solution dynamic, one that is neither spatio-graphical nor theoretical yet. In a dynamic-geometry world where everything moves, the point of reference may be redefined as well, as long as this use is supported by the software. Therefore, although no dependencies were created, as Sfard (personal communication) observed, the team's solution could be considered simply as a legitimate move, one that is situated in the environment.

A parallel progression can also be observed in the verification of the perpendicularity routine column. The team first felt the need to verify their solution, which was not explicitly requested in the instructions. Initially, this took a spatio-graphical form with Cheerios wanting to arrange the lines into a vertical-horizontal position. Then Cornflakes, who received help from Fruitloops, wanted to use the given construction of a perpendicular bisector as a protractor, turning the verification routine into one that is based on measurement. Eventually, Fruitloops, upon completing the construction, asked how they could be sure if the line was perpendicular. Cornflakes pointed at the visual appearance of the figure to convince Fruitloops. However, Fruitloops seemed to be looking for a verification routine that would go beyond the spatio-graphical. She even used the word 'proof' - though not necessarily in a deductive mathematical sense. This situation is quite contrary to the findings in the literature, as students' validation of a mathematical statement often takes the form of testing it against a few examples even at the more advanced levels (Chazan, 1993b; Coe and Ruthven, 1994). In the case of dynamic geometry, students often think that they can justify a claim by empirically checking the diagram (Laborde, 2004) - that is, by dragging.

The word "perpendicular" was first used by Cheerios in the first part of the task. She uttered the word only once, as if to revoice the instructions. Fruitloops, on the other hand, used the word throughout their problem-solving session. Her use of the word also represented a parallel advancement along with the production and verification routines. Initially the word signified a visual image of perpendicularity and was used to evaluate produced visual solutions. Later, however, her use of the word came to refer to a certain relationship between figures. Finally, it is reasonable to argue that the given example construction functioned an important role as the key visual mediator of the session. It was brought to the team's attention by Cornflakes, who first played with it randomly. But later she figured out a way to use it like a protractor, thus as a tool for verifying perpendicularity. This use may have led Fruitloops to view it as a straightedge that could in fact be used to draw the perpendicular. More importantly, however, it became the crucial visual mediator that triggered Fruitloops' use of circles, which led to framing the problem as a construction task.

\section{Conclusion}

We agree with Sfard (2008) that students cannot be expected to invent the meta-rules of mathematics on their own. However, our data indicate that an environment such as VMT may provide a context in which students can obtain a chance to engage in higher-level mathematical discourses. Thus, along with expert others, welldesigned virtual collaborative learning environments can provide a form of expert interaction that supports discourse development. In that regard, our findings support Sinclair and Moss (2012), who suggested that dynamic-geometry software could function as the discourse of experts. In our case, dynamic geometry was a component of the VMT software, which was built to support collaborative learning with a specific geometry curriculum (Stahl, 2013b). In 
addition to the dynamic geometry component, the curriculum and the collaborative interaction aspects of the VMT

environment also played a role in supporting students' discourse development. Thus, the VMT environment can be viewed as an effective material condition for learning geometry.

\section{References}

Chazan, D. (1993b). High school geometry students' justification for their views of empirical evidence and mathematical proof. Educational Studies in Mathematics, 24, 359-387.

Coe, R., \& Ruthven, K. (1994). Proof practices and constructs of advanced mathematics students. British Educational Research Journal, 20(1), 41-53.

Hoyles, C., \& Jones, K. (1998). Proof in dynamic geometry contexts. In C. Mammana \& V. Villani (Eds.), Perspectives on the teaching of geometry for the 21st century (pp. 121-128). Dordrecht: Kluwer.

Jones, K. (2000). Providing a foundation for deductive reasoning: Students' interpretations when using dynamic geometry software and their evolving mathematical explanations. Educational Studies in Mathematics, 44, $55-85$.

Laborde, C. (2004). The hidden role of diagrams in students' construction of meaning in geometry. In J. Kilpatrick, C. Hoyles, \& O. Skovsmose (Eds.), Meaning in mathematics education (pp. 1-21). Dordrecht: Kluwer Academic Publishers.

Marrades, R., \& Gutierrez, A. (2000). Proofs produced by secondary school students learning geometry in a dynamic computer environment. Educational Studies in Mathematics, 44, 87-125.

Sfard, A. (2008). Thinking as communicating: Human development, the growth of discourses, and mathematizing. Cambridge, MA: MIT Press.

Sinclair, N. \& Moss, J. (2012). The more it changes, the more it becomes the same: The development of the routine of shape identification in dynamic geometry environment. International Journal of Educational Research, 51-52, 28-44.

Stahl, G. (2013a). Translating Euclid: Creating a human-centered mathematics. San Rafael, CA: Morgan \& Claypool Publishers.

Stahl, G. (2013b). Explore dynamic geometry together. Retrieved July 17, 2014, from http://GerryStahl.net/elibrary/topics/explore.pdf . 